Researches in Mathematics and Mechanics. - 2020. - V. 25, Is. 1(35). - P. 16-30

UDC 536.24

O. F. Kryvyi, O. Yu. Morozov

\title{
THE FUNDAMENTAL SOLUTION OF THE PROBLEM OF THERMOELASTICITY FOR A PIECEWISE HOMOGENEOUS TRANSVERSELY ISOTROPIC ELASTIC SPACE
}

The problem of constructing fundamental solutions to the thermoelasticity problem for a piecewise-homogeneous transversely isotropic space is reduced to the matrix Riemann problem in the space of generalized slow growth functions. As a result of the solution of which, were obtained expressions in explicit form for the components of the vector of the fundamental solution of the heat conduction problem, as well as simple representations for the components of the stress tensor and the displacement vector in plane of connection of transversely isotropic elastic half-spaces containing concentrated stationary heat sources. The temperature distribution is investigated depending on the thermophysical characteristics of the half-space materials.

MSC: 74B05, 74H05, 74J20.

Key words: fundamental solutions, matrix Riemann problem,transversely isotropic inhomogeneous space, generalized functions.

DOI: $X X X X$.

\section{INTRODUCTION}

The study of stress concentration in the vicinity of interfacial and internal defects such as cracks or inclusions in thermoelastic fields is of great practical importance. Many works have been devoted to this problem for various environments. In particular, in [1-2], the problems of stationary thermoelasticity for bodies with a heat-penetrating disc inclusion, between whose surfaces there is an imperfect thermal contact, as well as problems with a thin heat-active disc inclusion are considered.

The problem is reduced to hypersingular integral equations of the first and second kind, for which exact solutions are obtained.In [3-9] non-axisymmetric problems of elasticity and thermoelasticity for piecewise-homogeneous transversely isotropic spaces containing interfacial stress concentrators, such as cracks or rigid inclusions, using the method of singular integral relations (SIR) [10] reduced to systems of two-dimensional singular integral equations (SIR) 
and proposed a method for their solution. A similar approach was applied in [11-15] to solving problems of interfacial and internal defects in piecewise homogeneous anisotropic media.

In the mathematical formulation and solution of such problems about defects, it is necessary to set the boundary conditions on the defect itself, such as stress on the crack edges or displacement at the inclusion. Since in thy physical formulation of the problems from determining the stress and displacement fields in the vicinity of the stress concentrators, known the stresses or displacements at the boundary of the region, at some interior points or at infinity (for unbounded bodies), then the determination of the boundary conditions on the defect is a separate problem.

Within the framework of the linear theory of thermoelasticity, to solve this problem, it is necessary to know the distribution of the temperature, stress and displacement fields in the corresponding piecewise homogeneous bodies without defects in the presence of volumetric forces and concentrated heat sources.

In particular, for piecewise homogeneous isotropic and transversally isotropic spaces, such solutions are given, respectively, in [16] and [17].Green's functions for piecewise homogeneous transversally isotropic spaces in the presence of a concentrated heat source and in the absence of thermal diffusion were constructed in [18], and in the presence of thermal diffusion - in [19]. In [20, 21], Green's functions for a layered thermal environment were constructed.

An effective method for solving this problem is the method of fundamental solutions in the space $\Im^{\prime}\left(\mathbb{R}^{3}\right)$ of generalized functions of slow growth. In particular, in [14], the problem of constructing fundamental solutions for piecewise homogeneous two-dimensional anisotropic media is reduced to the matrix Riemann problem with respect to some variables in the space $\Im^{\prime}\left(\mathbb{R}^{3}\right)$ and suggested an approach to its solution. In this work, this approach is generalized to construct in an explicit analytical form fundamental solutions to the problem of heat conduction and thermoelasticity for a piecewise-homogeneous transversely isotropic space, which made it possible to study the temperature distribution and obtain how the temperature affects the distribution of stresses and displacements in the plane of joining materials. 


\section{Main Results}

1. Statement of the problem. Let stationary heat sources, concentrated in some regions of dimension $\mathrm{n}(\mathrm{n}=0,1,2,3)$, act in an inhomogeneous space composed of two different transversely isotopic half-spaces, completely linked in the plane $z=0$.

The thermoelastic state of space is described by the vector

$$
\mathbf{v}=\left\{v_{k}(x, y, z)\right\}_{k=\overline{1,9}}=\left\{\sigma_{x}, \sigma_{y}, \sigma_{z}, \tau_{y z}, \tau_{x z}, \tau_{x y}, u, v, w\right\}
$$

Based on the equilibrium equations and the generalized Hooke's law, and also taking into account the Duhamel-Neumann relation with respect to the components of the vector $\mathbf{v}$, in the space of generalized functions of slow growth $\Im^{\prime}\left(\mathbb{R}^{3}\right)$ we write the following boundary value problem

$$
\begin{gathered}
\mathbf{D}\left[z, \partial_{1}, \partial_{2}, \partial_{3}\right] \mathbf{v}=\mathbf{F}, \quad \mathbf{v}, \mathbf{F} \in \Im^{\prime}\left(\mathbb{R}^{3}\right), \\
v_{k}(x, y,+0)=v_{k}(x, y,-0), k=\overline{1,9}, k \neq 1,2,6 \\
\left.v_{k}(x, y, x)\right|_{(x, y, z) \rightarrow \infty}=0, \quad(k=\overline{1,9}),
\end{gathered}
$$

Here we use the notation

$$
\mathbf{D}=\left\|\begin{array}{cc}
\mathbf{D}_{0} & \mathbf{O}_{3 \times 3} \\
-\mathbf{S} & \mathbf{D}_{0}^{T}
\end{array}\right\|, \mathbf{F}^{T}=\left\|0,0,0, \beta_{1}, \beta_{2}, \beta_{3}, 0,0,0\right\|, \mathbf{S}=\left\|\begin{array}{cc}
\mathbf{S}_{1} & \mathbf{O}_{3 \times 3} \\
\mathbf{O}_{3 \times 3} & \mathbf{S}_{2}
\end{array}\right\|
$$

$\mathbf{D}_{0}=\left\|\begin{array}{cccccc}\partial_{1} & 0 & 0 & 0 & \partial_{3} & \partial_{2} \\ 0 & \partial_{2} & 0 & \partial_{3} & 0 & \partial_{1} \\ 0 & 0 & \partial_{3} & \partial_{2} & \partial_{1} & 0\end{array}\right\|, \mathbf{S}_{1}=\left\|\begin{array}{ccc}s_{11} & s_{12} & s_{13} \\ s_{21} & s_{11} & s_{13} \\ s_{13} & s_{13} & s_{33}\end{array}\right\|, \mathbf{S}_{2}=\left\|\begin{array}{ccc}s_{44} & 0 & 0 \\ 0 & s_{44} & 0 \\ 0 & 0 & s_{66}\end{array}\right\|$ $\partial_{1}=\frac{\partial}{\partial x}, \partial_{2}=\frac{\partial}{\partial y}, \partial_{3}=\frac{\partial}{\partial z}, s_{k j}=\theta(z) s_{k j}^{+}+\theta(-z) s_{k j}^{-}, s_{k j}^{p m}-$ the coefficients of the generalized Hooke's law, respectively, for the upper $z>0$ and lower $z<0$ half-spaces; $\mathbf{O}_{3 \times 3}-$ zero matrix of dimension $3 \times 3, \beta_{k}=\theta(z) \beta_{k}^{+}+$ $\theta(-z) \beta_{k}^{-}, \beta_{k}^{ \pm}$- thermal expansion coefficients , $T$ - temperature concentrated heat source.

\section{Construction of the fundamental solution of the heat conduc- tion problem.}


We introduce the following notation $\mathbf{w}=\left\{w_{k}\right\}_{k=\overline{1,4}}$, where functions $w_{k}(x, y, z) \in \Im^{\prime}\left(\mathbb{R}^{3}\right)$ there are components of the system of fundamental solutions, that is, solutions that satisfy the following system of boundary value problems

$$
\begin{gathered}
\mathbf{D}\left[z, \partial_{1}, \partial_{2}, \partial_{3}\right] \mathbf{w}=\mathbf{f}^{0}, \quad \mathbf{w}_{j}, \mathbf{f}^{0} \in \Im^{\prime}\left(\mathbb{R}^{3}\right), \\
w_{4}(x, y,+0)=w_{4}(x, y,-0), \lambda_{3}^{+} \partial_{3} w_{4}(x, y,+0)=\lambda_{3}^{-} \partial_{3} w_{4}(x, y,-0)
\end{gathered}
$$

where

$$
\mathbf{D}=\left(\begin{array}{cccc}
\lambda_{1}^{-1} & 0 & 0 & \partial_{1} \\
0 & \lambda_{2}^{-1} & 0 & \partial_{2} \\
0 & 0 & \lambda_{3}^{-1} & \partial_{3} \\
\partial_{1} & \partial_{2} & \partial_{3} & 0
\end{array}\right), \mathbf{f}^{0}=\left\{-\delta_{k 4} \delta\left(x-x_{0}, y-y_{0}, z-z_{0}\right)\right\}_{k=\overline{1,4}}
$$

$\delta_{k j}$-Kronecker symbol, $\lambda_{i}=\lambda_{i}^{+} \theta\left(x_{3}\right)+\lambda_{i}^{-} \theta\left(-x_{3}\right), i=\overline{1,3}, \lambda_{i}^{ \pm}$-thermal conductivity coefficients for the upper $z>0$ and lower $z<0$ half-spaces, respectively.

Vector components $\mathbf{w}$ represented as $w_{k}=\theta(z) w_{k}+\theta(-z) w_{k}=w_{k}^{+}+w_{k}^{-}$, де $w_{k}^{ \pm} \in \Im^{\prime}\left(\mathbb{R}_{ \pm}^{3}\right), \mathbb{R}_{ \pm}^{3}=\mathbb{R}^{2} \times \mathbb{R}_{ \pm}$and apply to the matrix equation (5) the operator of the three-dimensional Fourier transform $F_{3}$ from $\Im^{\prime}\left(\mathbb{R}^{3}\right)$. Then, considering the conditions (6) and results of works [10-13,26,27], relatively $\mathrm{W}_{k}^{ \pm}\left(\alpha_{1}, \alpha_{2}, \alpha_{3}\right)=\mathrm{F}_{3}\left[w_{k}^{ \pm}\right] \in \Im^{\prime}\left(\mathbb{R}^{3}\right)$ we obtain the following matrix equation

$$
\begin{gathered}
\mathbf{B}^{+} \mathbf{W}^{+}=\mathbf{B}^{-} \mathbf{W}^{-}+\mathbf{F}^{0}, \mathbf{W}_{j}^{ \pm}, \mathbf{F}_{j}^{0} \in \Im^{\prime}\left(\mathbb{R}^{3}\right), j=\overline{1,4} . \\
\mathbf{W}^{ \pm}=\left\{\mathbf{W}_{k}^{ \pm}\right\}_{k=1}^{4}, \quad \mathbf{B}^{ \pm}=\mathbf{D}\left[ \pm 0,-i \alpha_{1},-i \alpha_{2},-i \alpha_{3}\right] \\
\mathbf{F}_{j}^{0}=\left\{\delta_{k 4} e^{i \alpha_{1} x_{0}+i \alpha_{2} y_{0}+i \alpha_{3} z_{0}}\right\}_{k=1}^{4}, \\
\mathbf{D}\left[ \pm 0,-i \alpha_{1},-i \alpha_{2},-i \alpha_{3}\right]=\left(\begin{array}{cccc}
\lambda_{1, \pm}^{-1} & 0 & 0 & \left(-i \alpha_{1}\right) \\
0 & \lambda_{1, \pm}^{-1} & 0 & \left(-i \alpha_{2}\right) \\
0 & 0 & \lambda_{3, \pm}^{-1} & \left(-i \alpha_{3}\right) \\
\left(-i \alpha_{1}\right) & \left(-i \alpha_{2}\right) & \left(-i \alpha_{3}\right) & 0
\end{array}\right) .
\end{gathered}
$$

Function $w_{k j}^{ \pm} \in \Im^{\prime}\left(\mathbb{R}_{ \pm}^{3}\right)$ admit an analytical representation $[22,26,27]$ on the variable $\alpha_{3}$, therefore, matrix equation (1.7) is a boundary condition for the matrix Riemann problem in the variable $\alpha_{3}$. 
Given the properties of generalized functions and applying the methodology of [10-14,26,27], the boundary conditions (1.7) can be written as

$$
\mathbf{B}^{ \pm} \mathbf{W}^{ \pm}=\mathbf{F}^{ \pm}, \mathbf{W}^{ \pm}, \mathbf{F}^{ \pm} \in \Im^{\prime}\left(\mathbb{R}^{3}\right)
$$

where

$$
\begin{aligned}
\mathbf{F}_{j}^{ \pm} & =\left\{f_{k}^{ \pm}\right\}_{k=\overline{1,4}}, & f_{k}^{ \pm} & =\theta\left( \pm z_{0}\right) e_{0}^{ \pm} \delta_{k 4} \mp \frac{1}{2} \chi_{k}, \\
\chi & =\left\{\chi_{k}\right\}_{k=\overline{1,4}} \in \Im^{\prime}\left(\mathbb{R}^{2}\right), & \chi_{k} & =0, k=1,2 .
\end{aligned}
$$

$\chi_{k}\left(\alpha_{1}, \alpha_{2}\right)$ - unknown functions from $\Im^{\prime}\left(\mathbb{R}^{2}\right)$ for determine of which, we use conditions (7) in Fourier transforms.

Directly from equations (1.8) we obtain $\mathbf{W}^{ \pm}=\mathbf{B}_{ \pm}^{-1} \mathbf{F}^{ \pm}$, where $\mathbf{B}_{ \pm}^{-1}=$ $\left\{b_{k}^{*, \pm}\right\}_{k=\overline{1,4}}$. After applying the inverse Fourier transform, the components of the vectors $\mathbf{w}^{ \pm}=\left\{w_{k}^{ \pm}\right\}_{k=\overline{1,4}}=\mathrm{F}_{3}^{-1}\left[\mathbf{W}^{ \pm}\right]$will be presented as:

$$
\begin{aligned}
& w_{1}^{+}=\frac{\left(x-x_{0}\right)}{2 r}\left\{\theta\left(z_{0}\right)\left(\frac{m_{11}^{+}\left(r_{0}^{2}+\left(\xi_{n}^{+}\left|z-z_{0}\right|\right)^{2}\right)^{-1 / 2}}{\left(\xi_{n}^{+}\left|z-z_{0}\right|+\sqrt{r_{0}^{2}+\left(\xi_{n}^{+}\left|z-z_{0}\right|\right)^{2}}\right)}-\frac{m_{12}^{+}\left(r_{0}^{2}+\left(\xi_{0}^{+}\left(z+z_{0}\right)\right)^{2}\right)^{-1 / 2}}{\left(\xi_{0}^{+}\left|z+z_{0}\right|+\sqrt{r_{0}^{2}+\left(\xi_{0}^{+}\left(z+z_{0}\right)\right)^{2}}\right)}\right)+\right. \\
& \left.+\theta\left(-z_{0}\right) \frac{m_{13}^{+}\left(r_{0}^{2}+\left(\xi_{0}^{-} z_{0}-\xi_{0}^{+} z\right)^{2}\right)^{-1 / 2}}{\left(\left|\xi_{0}^{-} z_{0}-\xi_{0}^{+} z\right|+\sqrt{r_{0}^{2}+\left(\xi_{0}^{-} z_{0}-\xi_{0}^{+} z\right)^{2}}\right.}\right\}, \\
& w_{1}^{-}=\frac{\left(x-x_{0}\right)}{2 r}\left\{\theta\left(-z_{0}\right)\left(\frac{m_{11}^{-}\left(r_{0}^{2}+\left(\xi_{n}^{-}\left|z-z_{0}\right|\right)^{2}\right)^{-1 / 2}}{\left(\xi_{n}^{-}\left|z-z_{0}\right|+\sqrt{r_{0}^{2}+\left(\xi_{n}^{-}\left|z-z_{0}\right|\right)^{2}}\right)}-\frac{m_{12}^{-}\left(r_{0}^{2}+\left(\xi_{0}^{-}\left(z+z_{0}\right)\right)^{2}\right)^{-1 / 2}}{\left(\xi_{0}^{-}\left|z+z_{0}\right|+\sqrt{r_{0}^{2}+\left(\xi_{0}^{-}\left(z+z_{0}\right)\right)^{2}}\right)}\right)-\right. \\
& \left.-\theta\left(z_{0}\right) \frac{m_{13}^{-}\left(r_{0}^{2}+\left(\xi_{0}^{-} z-\xi_{0}^{+} z_{0}\right)^{2}\right)^{-1 / 2}}{\left(\left|\xi_{0}^{-} z-\xi_{0}^{+} z_{0}\right|+\sqrt{r_{0}^{2}+\left(\xi_{0}^{-} z-\xi_{0}^{+} z_{0}\right)^{2}}\right.}\right\}, \\
& w_{2}^{+}=\frac{\left(y-y_{0}\right)}{2 r}\left\{\theta\left(z_{0}\right)\left(\frac{m_{11}^{+}\left(r_{0}^{2}+\left(\xi_{n}^{+}\left|z-z_{0}\right|\right)^{2}\right)^{-1 / 2}}{\left(\xi_{n}^{+}\left|z-z_{0}\right|+\sqrt{r_{0}^{2}+\left(\xi_{n}^{+}\left|z-z_{0}\right|\right)^{2}}\right)}-\frac{m_{12}^{+}\left(r_{0}^{2}+\left(\xi_{0}^{+}\left(z+z_{0}\right)\right)^{2}\right)^{-1 / 2}}{\left(\xi_{0}^{+}\left|z+z_{0}\right|+\sqrt{r_{0}^{2}+\left(\xi_{0}^{+}\left(z+z_{0}\right)\right)^{2}}\right)}\right)+\right. \\
& \left.+\theta\left(-z_{0}\right) \frac{m_{13}^{+}\left(r_{0}^{2}+\left(\xi_{0}^{-} z_{0}-\xi_{0}^{+} z\right)^{2}\right)^{-1 / 2}}{\left(\left|\xi_{0}^{-} z_{0}-\xi_{0}^{+} z\right|+\sqrt{r_{0}^{2}+\left(\xi_{0}^{-} z_{0}-\xi_{0}^{+} z\right)^{2}}\right)}\right\}, \\
& w_{2}^{-}=\frac{\left(y-y_{0}\right)}{2 r}\left\{\theta\left(-z_{0}\right)\left(\frac{m_{11}^{-}\left(r_{0}^{2}+\left(\xi_{n}^{-}\left|z-z_{0}\right|\right)^{2}\right)^{-1 / 2}}{\left(\xi_{n}^{-}\left|z-z_{0}\right|+\sqrt{r_{0}^{2}+\left(\xi_{n}^{-}\left|z-z_{0}\right|\right)^{2}}\right)}-\frac{m_{12}^{-}\left(r_{0}^{2}+\left(\xi_{0}^{-}\left(z+z_{0}\right)\right)^{2}\right)^{-1 / 2}}{\left(\xi_{0}^{-}\left|z+z_{0}\right|+\sqrt{r_{0}^{2}+\left(\xi_{0}^{-}\left(z+z_{0}\right)\right)^{2}}\right)}\right)-\right. \\
& \left.-\theta\left(z_{0}\right) \frac{m_{13}^{-}\left(r_{0}^{2}+\left(\xi_{0}^{-} z-\xi_{0}^{+} z_{0}\right)^{2}\right)^{-1 / 2}}{\left(\left|\xi_{0}^{-} z-\xi_{0}^{+} z_{0}\right|+\sqrt{r_{0}^{2}+\left(\xi_{0}^{-} z-\xi_{0}^{+} z_{0}\right)^{2}}\right)}\right\}, \\
& w_{3}^{+}=\left\{\theta\left(z_{0}\right)\left(m_{21}^{+} \frac{\operatorname{sign}\left(z-z_{0}\right) \xi_{0}^{+}\left|z-z_{0}\right|}{\left(r_{0}^{2}+\left(\xi_{0}^{+}\left|z-z_{0}\right|\right)^{2}\right)^{3 / 2}}-\frac{m_{22}^{+} \xi_{0}^{+}\left(z+z_{0}\right)}{\left(r_{0}^{2}+\left(\xi_{0}^{+}\left(z+z_{0}\right)\right)^{2}\right)^{3 / 2}}\right)-\right. \\
& \left.-\theta\left(-z_{0}\right) \frac{m_{23}^{+}\left|\xi_{0}^{+} z-\xi_{0}^{-} z_{0}\right|}{\left(r_{0}^{2}+\left(\xi_{0}^{+} z-\xi_{0}^{-} z_{0}\right)^{2}\right)^{3 / 2}}\right\},
\end{aligned}
$$




$$
\begin{gathered}
w_{3}^{-}=\left\{\theta\left(-z_{0}\right)\left(m_{21}^{-} \frac{\operatorname{sign}\left(z-z_{0}\right) \xi_{0}^{-}\left|z-z_{0}\right|}{\left(r_{0}^{2}+\left(\xi_{0}^{-}\left|z-z_{0}\right|\right)^{2}\right)^{3 / 2}}-\frac{m_{22}^{+} \xi_{0}^{+}\left(z+z_{0}\right)}{\left(r_{0}^{2}+\left(\xi_{0}^{-}\left(z+z_{0}\right)\right)^{2}\right)^{3 / 2}}\right)+\right. \\
\left.+\theta\left(-z_{0}\right) \frac{m_{23}^{-} \xi_{0}^{+} z_{0}-\xi_{0}^{-} r z \mid}{\left(r_{0}^{2}+\left(\xi_{0}^{+} z_{0}-\xi_{0}^{-} r z\right)^{2}\right)^{3 / 2}}\right\}, \\
w_{4}^{+}=\left\{\theta\left(z_{0}\right)\left(\frac{m_{31}^{+}}{\sqrt{r_{0}^{2}+\left(\xi_{0}^{+}\left|z-z_{0}\right|\right)^{2}}}-\frac{m_{32}^{+}}{\sqrt{r_{0}^{2}+\left(\xi_{0}^{+}\left(z+z_{0}\right)\right)^{2}}}\right)+\right. \\
\left.+\theta\left(-z_{0}\right) \frac{m_{33}^{+}}{\sqrt{r_{0}^{2}+\left(\xi_{0}^{+} z-\xi_{0}^{-} z_{0}\right)^{2}}}\right\}, \\
w_{4}^{-}=\left\{\theta\left(-z_{0}\right)\left(\frac{m_{31}^{-}}{\sqrt{r_{0}^{2}+\left(\xi_{0}^{-}\left|z-z_{0}\right|\right)^{2}}}+\frac{m_{32}^{-}}{\sqrt{r_{0}^{2}+\left(\xi_{0}^{-}\left(z+z_{0}\right)\right)^{2}}}\right)-\right. \\
\left.-\theta\left(z_{0}\right) \frac{m_{33}^{-}}{\sqrt{r_{0}^{2}+\left(\xi_{0}^{-} z-\xi_{0}^{+} z_{0}\right)^{2}}}\right\}
\end{gathered}
$$

where

$$
\begin{gathered}
m_{11}^{ \pm}=\frac{\lambda_{1}^{ \pm}}{\xi_{0}^{ \pm}}, \quad m_{12}^{ \pm}=\lambda_{1}^{ \pm}\left(\lambda_{3}^{ \pm} m_{1}^{ \pm} \pm \frac{m_{2}^{ \pm}}{\xi_{0}^{ \pm}}\right), \quad m_{13}^{ \pm}=\lambda_{1}^{ \pm}\left(\lambda_{3}^{ \pm} m_{1}^{\mp} \pm \frac{m_{2}^{\mp}}{\xi_{0}^{ \pm}}\right), \\
m_{21}^{ \pm}=1, \quad m_{22}^{ \pm}=\frac{\lambda_{1}^{ \pm} \lambda_{3}^{+}}{\xi_{0}^{ \pm}} m_{1}^{ \pm} \pm \lambda_{3}^{ \pm} m_{2}^{ \pm}, \quad m_{23}^{ \pm}=\frac{\lambda_{1}^{ \pm} \lambda_{3}^{+}}{\xi_{0}^{ \pm}} m_{1}^{\mp} \pm \lambda_{3}^{ \pm} m_{2}^{\mp} \\
m_{31}^{ \pm}=\frac{1}{\lambda_{3}^{ \pm} \xi_{0}^{ \pm}}, \quad m_{32}^{ \pm}=\lambda_{3}^{ \pm} m_{1}^{ \pm} \pm \frac{m_{2}^{ \pm}}{\xi_{0}^{ \pm}}, \quad m_{33}^{ \pm}=\lambda_{3}^{ \pm} m_{1}^{\mp}+\frac{m_{2}^{\mp}}{\xi_{0}^{ \pm}}
\end{gathered}
$$

Sought temperature $T$ we get like this $T=w_{4} * Q$.

3. Construction of a fundamental solution to the problem of thermoelasticity. We apply to the matrix equation (2) the operator of the three-dimensional Fourier transform $\mathrm{F}_{3}$ is $\Im^{\prime}\left(\mathbb{R}^{3}\right)$, given the following representation for vector components $\mathbf{v}: v_{k}=\theta(z) v_{k}+\theta(-z) v_{k}=v_{k}^{+}+v_{k}^{-}$, were $v_{k}^{ \pm} \in \Im^{\prime}\left(\mathbb{R}_{ \pm}^{3}\right), \mathbb{R}_{ \pm}^{3}=\mathbb{R}^{2} \times \mathbb{R}_{ \pm}$. Then, considering the conditions (3) and the results of $[10-14,26,27]$, with respect to $\mathrm{V}_{k}^{ \pm}\left(\alpha_{1}, \alpha_{2}, \alpha_{3}\right)=\mathrm{F}_{3}\left[v_{k}^{ \pm}\right] \in \Im^{\prime}\left(\mathbb{R}^{3}\right)$ and also that the functions $\mathrm{V}_{k}^{ \pm} \in \Im^{\prime}\left(\mathbb{R}_{ \pm}^{3}\right)$ admit an analytical representation $[22,26,27]$ in the variable $\alpha_{3}$ we obtain the following matrix equation

$$
\mathbf{M}_{ \pm} \mathbf{V}^{ \pm}=\mathbf{F}^{ \pm}, \mathbf{W}^{ \pm}, \mathbf{F}^{ \pm} \in \Im^{\prime}\left(\mathbb{R}^{3}\right)
$$

where

$$
\begin{aligned}
\mathbf{M}_{ \pm} & =\mathbf{D}\left[ \pm 0,-i \alpha_{1},-i \alpha_{2},-i \alpha_{3}\right], \quad \mathbf{F}_{j}^{ \pm}=\left\{f_{k}^{ \pm}\right\}_{k=\overline{1,4}} \\
f_{k}^{ \pm} & =\mp \frac{1}{2} \chi_{k}, \quad k=\overline{1,9}, \quad k \neq 3,4,5, \\
f_{k}^{ \pm} & =\beta_{k-2}^{ \pm} T^{ \pm} \mp \frac{1}{2} \chi_{k}, \quad k=3,4,5, \\
\chi & =\left\{\chi_{k}\right\}_{k=1,4} \in \Im^{\prime}\left(\mathbb{R}^{2}\right), \quad \chi_{k}=0, \quad k=4,5,9
\end{aligned}
$$


$\chi_{k}\left(\alpha_{1}, \alpha_{2}\right)$ - unknown functions from $\Im^{\prime}\left(\mathbb{R}^{2}\right)$ for determine which, we need to use conditions (3) after the Fourier-transformed.

We represent the sought functions as

$$
\begin{gathered}
\mathrm{V}_{7 j}^{ \pm}=-\left(-i \alpha_{2}\right) \Psi_{1 j}^{ \pm}-\left(-i \alpha_{1}\right) \Psi_{2 j}^{ \pm}, \mathrm{V}_{8 j}^{ \pm}=\left(-i \alpha_{1}\right) \Psi_{1 j}^{ \pm}-\left(-i \alpha_{2}\right) \Psi_{2 j}^{ \pm}, \\
\mathrm{V}_{5 j}^{ \pm}=-\left(-i \alpha_{2}\right) \Upsilon_{1 j}^{ \pm}-\left(-i \alpha_{1}\right) \Upsilon_{2 j}^{ \pm}, \mathrm{V}_{4 j}^{ \pm}=\left(-i \alpha_{1}\right) \Upsilon_{1 j}^{ \pm}-\left(-i \alpha_{2}\right) \Upsilon_{2 j}^{ \pm}
\end{gathered}
$$

where $\Psi_{k}^{ \pm}, \Upsilon_{k}^{ \pm}(k=1,2)$ new unknown functions, then matrix equation (9) can be separated into two independent equations

$$
\mathbf{L}_{ \pm} \mathbf{V}^{(1), \pm}=\mathbf{F}_{1}^{ \pm}, \mathbf{G}_{ \pm} \mathbf{V}^{(2), \pm}=\mathbf{F}_{2}^{ \pm}
$$

where we use the notation

$$
\begin{aligned}
\mathbf{V}^{(1), \pm} & =\left\{\mathrm{V}_{k}^{(1), \pm}\right\}_{k=1,2}=\left\{\Upsilon_{1 j}^{ \pm}, \Psi_{1 j}^{ \pm}\right\}, \\
\mathbf{V}^{(2), \pm} & =\left\{\mathrm{V}_{k}^{(2), \pm}\right\}_{k=1,4}=\left\{\mathrm{W}_{3 j}^{ \pm}, \mathrm{T}_{2 j}^{ \pm}, \Psi_{2 j}^{ \pm}, \mathrm{W}_{9 j}^{ \pm}\right\} \\
\mathbf{F}_{j 1}^{ \pm} & =\left\{\left(-i \alpha_{2}\right) f_{1 j}^{ \pm}-\left(-i \alpha_{1}\right) f_{2 j}^{ \pm},\left(-i \alpha_{2}\right) f_{7 j}^{ \pm}-\left(-i \alpha_{1}\right) f_{8 j}^{ \pm}\right\}, r^{2}=\alpha_{1}^{2}+\alpha_{2}^{2}, \\
\mathbf{F}_{j 2}^{ \pm} & =\left\{f_{3 j}^{ \pm},\left(-i \alpha_{1}\right) f_{1 j}^{ \pm}+\left(-i \alpha_{2}\right) f_{2 j}^{ \pm},\left(-i \alpha_{2}\right) f_{8 j}^{ \pm}+\left(-i \alpha_{1}\right) f_{7 j}^{ \pm}, f_{6 j}^{ \pm}\right\} \\
\mathbf{G}_{ \pm} & =\left\{g_{k j}^{ \pm}\right\}_{k, j=1,4}, g_{11}^{ \pm}=g_{44}^{ \pm}=\left(-i \alpha_{3}\right), g_{12}^{ \pm}=r^{2}, g_{22}^{ \pm}=g_{33}^{ \pm}=\left(-i \alpha_{3}\right) r^{2}, \\
g_{k j}^{ \pm} & =g_{j k}^{ \pm}=0, \quad k=1,2, \quad j=3,4, \\
g_{21}^{ \pm} & =-\frac{c_{13}^{ \pm}}{c_{33}^{ \pm}} g_{12}^{ \pm}, \quad g_{23}^{ \pm}=-\frac{c_{13}^{ \pm}+c_{13}^{ \pm}}{c_{33}^{ \pm}} r^{4}, \quad g_{32}^{ \pm}=-\frac{1}{c_{44}^{ \pm}} g_{12}^{ \pm}, \\
g_{34}^{ \pm} & =-g_{12}^{ \pm}, \quad g_{41}^{ \pm}=-\frac{1}{c_{33}^{ \pm}}, \quad g_{43}^{ \pm}=\frac{c_{13}^{ \pm}}{c_{33}^{ \pm}} g_{12}^{ \pm}, \quad \mathbf{L}_{ \pm}=\left\{l_{k j}^{ \pm}\right\}_{k, j=1,2}, \\
l_{11}^{ \pm} & =\left(-i \alpha_{3}\right) r^{-2}, \quad l_{22}^{ \pm}=\left(-i \alpha_{3}\right) r^{2}, \quad l_{21}^{ \pm}=-r^{2} / c_{44}^{ \pm}, \quad l_{21}^{ \pm}=-c_{66} r^{4},
\end{aligned}
$$

Directly from equations (12) we obtain $\mathbf{V}^{(1), \pm}=\mathbf{L}_{ \pm}^{-1} \mathbf{F}_{j 1}^{ \pm}, \mathbf{V}^{(2), \pm}=\mathbf{G}_{ \pm}^{-1} \mathbf{F}_{j 2}^{ \pm}$, were $\mathbf{L}_{ \pm}^{-1}=\left\{l_{k j}^{*, \pm}\right\}_{k, j=1,2}, \mathbf{G}_{ \pm}^{-1}=\left\{g_{k j}^{*, \pm}\right\}_{i, j=\overline{1,4}}$. Further, using representations 
(11), (12) after applying the inverse Fourier transform, we obtain:

$$
\begin{aligned}
& \sigma_{3}=-\sum_{n=1}^{3}\left(\frac{R_{1, n}^{0}}{\sqrt{r_{0}^{2}+\left(\xi_{n}\left|z-z_{0}\right|\right)^{2}}}-\frac{\omega_{1, n}}{\sqrt{r_{0}^{2}+\left(\bar{\xi}_{n} z+\breve{\xi}_{0} z_{0}\right)^{2}}}\right) \\
& +\sum_{n=1}^{2} \sum_{m=1}^{3} \frac{\alpha_{1, n, m}}{\sqrt{r_{0}^{2}+\left(\widetilde{\xi}_{n} z+\breve{\xi}_{m} z_{0}\right)^{2}}} \\
& \sigma_{4}=\left(y-y_{0}\right)\left\{\sum_{n=1}^{3} \frac{R_{2, n}^{0}\left(r_{0}^{2}+\left(\xi_{n}\left|z-z_{0}\right|\right)^{2}\right)^{-1 / 2}}{\left(\xi_{n}\left|z-z_{0}\right|+\sqrt{r_{0}^{2}+\left(\xi_{n}\left|z-z_{0}\right|\right)^{2}}\right)}\right. \\
& -\sum_{n=1}^{3} \frac{\omega_{2, n}\left(r_{0}^{2}+\left(\widehat{\xi}_{n} z+\breve{\xi}_{0} z_{0}\right)^{2}\right)^{-1 / 2}}{\left(\widehat{\xi}_{n}|z|+\breve{\xi}_{0}\left|z_{0}\right|+\sqrt{r_{0}^{2}+\left(\widehat{\xi}_{n} z+\breve{\xi}_{0} z_{0}\right)^{2}}\right)} \\
& \left.-\sum_{n=1}^{2} \sum_{m=1}^{3} \frac{\alpha_{2, n, m}\left(r_{0}^{2}+\left(\widehat{\xi}_{n} z+\breve{\xi}_{m} z_{0}\right)^{2}\right)^{-1 / 2}}{\left(\widehat{\xi}_{n}|z|+\breve{\xi}_{m}\left|z_{0}\right|+\sqrt{r_{0}^{2}+\left(\widehat{\xi}_{n} z+\breve{\xi}_{m} z_{0}\right)^{2}}\right)}\right\}, \\
& \sigma_{5}=\left(x-x_{0}\right)\left\{\sum_{n=1}^{3} \frac{R_{2, n}^{0}\left(r_{0}^{2}+\left(\xi_{n}\left|z-z_{0}\right|\right)^{2}\right)^{-1 / 2}}{\left(\xi_{n}\left|z-z_{0}\right|+\sqrt{r_{0}^{2}+\left(\xi_{n}\left|z-z_{0}\right|\right)^{2}}\right)}\right. \\
& -\sum_{n=1}^{3} \frac{\omega_{2, n}\left(r_{0}^{2}+\left(\widehat{\xi}_{n} z+\breve{\xi}_{0} z_{0}\right)^{2}\right)^{-1 / 2}}{\left(\widehat{\xi}_{n}|z|+\breve{\xi}_{0}\left|z_{0}\right|+\sqrt{r_{0}^{2}+\left(\widehat{\xi}_{n} z+\breve{\xi}_{0} z_{0}\right)^{2}}\right)} \\
& \left.-\sum_{n=1}^{2} \sum_{m=1}^{3} \frac{\alpha_{2, n, m}\left(r_{0}^{2}+\left(\widehat{\xi}_{n} z+\breve{\xi}_{m} z_{0}\right)^{2}\right)^{-1 / 2}}{\left(\widehat{\xi}_{n}|z|+\breve{\xi}_{m}\left|z_{0}\right|+\sqrt{\left.r_{0}^{2}+\left(\widetilde{\xi}_{n} z+\breve{\xi}_{m} z_{0}\right)^{2}\right)}\right.}\right\}, \\
& u_{1}=\left(x-x_{0}\right)\left\{\sum_{n=1}^{3} \frac{R_{3, n}^{0}}{\left(\xi_{n}\left|z-z_{0}\right|+\sqrt{r_{0}^{2}+\left(\xi_{n}\left|z-z_{0}\right|\right)^{2}}\right)}\right. \\
& -\sum_{n=1}^{3} \frac{\omega_{3, n}}{\left(\widehat{\xi}_{n}|z|+\breve{\xi}_{0}\left|z_{0}\right|+\sqrt{r_{0}^{2}+\left(\widehat{\xi}_{n} z+\breve{\xi}_{0} z_{0}\right)^{2}}\right)} \\
& \left.-\sum_{n=1}^{2} \sum_{m=1}^{3} \frac{\alpha_{3, n, m}}{\left(\widehat{\xi}_{n}|z|+\breve{\xi}_{m}\left|z_{0}\right|+\sqrt{r_{0}^{2}+\left(\widehat{\xi}_{n} z+\breve{\xi}_{m} z_{0}\right)^{2}}\right)}\right\} \text {, } \\
& u_{2}=\left(y-y_{0}\right)\left\{\sum_{n=1}^{3} \frac{R_{3, n}^{0}}{\left(\xi_{n}\left|z-z_{0}\right|+\sqrt{r_{0}^{2}+\left(\xi_{n}\left|z-z_{0}\right|\right)^{2}}\right)}\right.
\end{aligned}
$$




$$
\begin{aligned}
& -\sum_{n=1}^{3} \frac{\omega_{3, n}}{\left(\bar{\xi}_{n}|z|+\breve{\xi}_{0}\left|z_{0}\right|+\sqrt{r_{0}^{2}+\left(\bar{\xi}_{n} z+\breve{\xi}_{0} z_{0}\right)^{2}}\right)} \\
& \left.-\sum_{n=1}^{2} \sum_{m=1}^{3} \frac{\alpha_{3, n, m}}{\left(\bar{\xi}_{n}|z|+\breve{\xi}_{m}\left|z_{0}\right|+\sqrt{r_{0}^{2}+\left(\bar{\xi}_{n} z+\breve{\xi}_{m} z_{0}\right)^{2}}\right)}\right\}, \\
u_{3}= & -\sum_{n=1}^{3} R_{4, n}^{0}\left(\ln \frac{c}{2}+\ln \left(\left|z-z_{0}\right| \xi_{n}^{+}+\sqrt{r_{0}^{2}+\left(\xi_{n}\left|z-z_{0}\right|\right)^{2}}\right)\right) \\
& +\sum_{n=1}^{3} \omega_{4, n}\left(\ln \frac{c}{2}+\ln \left(\left(\widetilde{\xi}_{n}|z|+\breve{\xi}_{0}\left|z_{0}\right|\right)+\sqrt{r_{0}^{2}+\left(\widehat{\xi}_{n} z+\breve{\xi}_{0} z_{0}\right)^{2}}\right)\right) \\
& +\sum_{n=1}^{2} \sum_{m=1}^{3} \alpha_{4, n, m}\left(\ln \frac{c}{2}+\ln \left(\left(\widehat{\xi}_{n}|z|+\breve{\xi}_{m}\left|z_{0}\right|\right)+\sqrt{r_{0}^{2}+\left(\widehat{\xi}_{n} z+\breve{\xi}_{m} z_{0}\right)^{2}}\right)\right),
\end{aligned}
$$

were

$$
\begin{gathered}
q_{j}^{ \pm}\left(\alpha_{3}, r\right)=g_{j 2}^{*, \pm}\left(\alpha_{3}, r\right) \gamma_{1}^{ \pm} r^{2}+g_{j 4}^{*, \pm}\left(\alpha_{3}, r\right) \gamma_{2}^{ \pm}, j=\overline{1,4} \\
R_{1, n}^{0,+}=\frac{q_{1}^{+}\left(-i \xi_{n}^{+}, 1\right) \tau^{++}\left(-i \xi_{n}^{+}, 1\right)}{\xi_{n}^{+} h_{n}^{+}}, \bar{R}_{1, n}^{0,+}=\frac{q_{1}^{+}\left(i \xi_{n}^{+}, 1\right) \tau^{++}\left(i \xi_{n}^{+}, 1\right)}{\xi_{n}^{+} h_{n}^{+}} \\
\tau^{++}\left(\alpha_{3}, r\right)=\left(-i \alpha_{3}\right) \lambda_{3}^{+} m_{1}^{+}-r m_{2}^{+}, \tau^{-+}\left(\alpha_{3}, r\right)=\left(-i \alpha_{3}\right) \lambda_{3}^{-} m_{1}^{+}-r m_{2}^{+}, \\
\tau^{+-}\left(\alpha_{3}, r\right)=\left(-i \alpha_{3}\right) \lambda_{3}^{+} m_{1}^{-}-r m_{2}^{-}, \tilde{\beta}_{1, n}^{-+}=\frac{q_{1}^{-}\left(i \xi_{n}^{ \pm}, 1\right) \tau^{-+}\left(i \xi_{n}^{-}, 1\right)}{\xi_{n}^{-} h_{n}^{-}}, \\
\tilde{\beta}_{1, n}^{+-}=\frac{q_{1}^{+}\left(-i \xi_{n}^{+}, 1\right) \tau^{+-}\left(-i \xi_{n}^{+}, 1\right)}{\xi_{n}^{+} h_{n}^{+}}, \tilde{\beta}_{j, n}^{++}=\frac{q_{j}^{+}\left(-i \xi_{n}^{+}, 1\right) \tau^{++}\left(-i \xi_{n}, 1\right)}{\xi_{n}^{+} h_{n}^{+}}, j=\overline{1,4} \\
h_{n}^{ \pm}=\prod_{l=1, l \neq n}^{3}\left(\xi_{n}^{ \pm}\right)^{2}-\left(\xi_{l}^{ \pm}\right)^{2}, \tau^{--}\left(\alpha_{3}, r\right)=\left(-i \alpha_{3}\right) \lambda_{3}^{-} m_{1}^{-}-r m_{2}^{-}, \\
\tilde{\beta}_{1, n}^{--}=\frac{q_{1}^{-}\left(i \xi_{n}^{-}, 1\right) \tau^{--}\left(i \xi_{n}^{-}, 1\right)}{\xi_{n}^{-} h_{n}^{-}}, R_{j, k}^{ \pm}=\sum_{n=1}^{2} R_{j, k, n}^{*,+}, \beta_{j}^{ \pm \pm}=\sum_{n=1}^{3} \tilde{\beta}_{j, n}^{ \pm \pm}, \\
\beta_{j}^{ \pm \mp}=\sum_{n=1}^{3} \tilde{\beta}_{j, n}^{ \pm \mp}, \mathbf{A}_{0}^{-1}=\left\{a_{k j}^{*}\right\}_{k, j=} \overline{1,4}, \mathbf{A}_{0}=\left\{a_{k, j}\right\}_{k, j=} \overline{1,4}=\mathbf{N}^{+}+\overline{\mathbf{N}}^{-}, \\
\mu_{j}^{+}=\sum_{k=1}^{4} a_{j k}^{*} \beta_{k}^{+}, \mu_{j}^{-}=\sum_{k=1}^{4} a_{j k}^{*} \beta_{k}^{-}, \beta_{j}^{+}=\beta_{j}^{++}+\beta_{j}^{-+}, \beta_{j}^{-}=\beta_{j}^{--}+\beta_{j}^{+-} \\
\mathbf{N}^{ \pm}=\left\{R_{j, k}^{ \pm}\right\}_{k, j=\overline{1,4}}, \alpha_{j, m}^{+}=\sum_{k=1}^{4} a_{j k}^{*} \bar{R}_{k, n}^{0,+}, \alpha_{j, m}^{-}=\sum_{k=1} a_{j k}^{*} R_{k, m}^{0,-}, \\
4
\end{gathered}
$$




$$
\begin{aligned}
& \alpha_{j, n, m}^{++}=\sum_{k=1}^{4} R_{j, k, n}^{*,+} \alpha_{k, m}^{+}, \alpha_{j, n, m}^{+-}=\sum_{k=1}^{4} R_{j, k, n}^{*,+} \alpha_{k, m}^{+}, \mu_{j}^{++}=\sum_{k=1}^{4} R_{j, k, n}^{*,+} \mu_{j}^{+}, \\
& \mu_{j, n}^{+-}=\sum_{k=1}^{4} R_{j, k, n}^{*,+} \mu_{j}^{-}, n=1,2, \mu_{j, n}^{+-}=0, n=3, \alpha_{j, n, m}^{--}=\sum_{k=1}^{4} R_{j, k, n}^{*,--} \alpha_{k, m}^{+}, \\
& \alpha_{j, n, m}^{-+}=\sum_{k=1}^{4} R_{j, k, n}^{*,-} \alpha_{k, m}^{+}, \mu_{j, n}^{--}=\sum_{k=1}^{4} R_{j, k, n}^{*,-} \mu_{j}^{-}, \mu_{j, n}^{-+}=\sum_{k=1}^{4} R_{j, k, n}^{*,-} \mu_{j}^{+}, n=1,2 \text {, } \\
& \mu_{j, n}^{--}=\mu_{j, n}^{-+}=0, n=3, \tilde{\omega}_{j, n}^{ \pm \pm}=\tilde{\beta}_{j, n}^{ \pm \pm}-\mu_{j, n}^{ \pm \pm}, \tilde{\omega}_{j, n}^{ \pm \mp}=\tilde{\beta}_{j, n}^{ \pm \mp}-\mu_{j, n}^{ \pm \mp}, \\
& \stackrel{\stackrel{R}{R, n}}{0,+}_{j,} \theta\left(z-z_{0}\right) R_{j, n}^{0,+}+\theta\left(z_{0}-z\right) \bar{R}_{j, n}^{0,+}, \stackrel{\smile}{R}_{j, n}^{0,-}=\theta\left(z-z_{0}\right) R_{j, n}^{0,-}+\theta\left(z_{0}-z\right) \bar{R}_{j, n}^{0,-} \\
& \omega_{j, n}=-\theta\left(z, z_{0}\right) \tilde{\omega}_{1, n}^{++}+\theta\left(z,-z_{0}\right) \tilde{\omega}_{1, n}^{+-}+\theta\left(-z, z_{0}\right) \tilde{\omega}_{1, n}^{-+}-\theta\left(-z,-z_{0}\right) \tilde{\omega}_{1, n}^{--} \\
& \alpha_{j, n, m}=\theta\left(z, z_{0}\right) \alpha_{j, m, n}^{++}-\theta\left(z,-z_{0}\right) \alpha_{j, m, n}^{+-}+\theta\left(-z,-z_{0}\right) \alpha_{j, m, n}^{--}-\theta\left(-z, z_{0}\right) \alpha_{j, m, n}^{-+} \\
& \widehat{\xi}_{n}=\theta\left(z, z_{0}\right) \xi_{n}^{+}-\theta\left(z,-z_{0}\right) \xi_{n}^{+}+\theta\left(-z,-z_{0}\right) \xi_{n}^{-}-\theta\left(-z, z_{0}\right) \xi_{n}^{-} \\
& \widehat{\xi}_{0}=\theta\left(z, z_{0}\right) \xi_{0}^{+}-\theta\left(z,-z_{0}\right) \xi_{0}^{+}+\theta\left(-z,-z_{0}\right) \xi_{0}^{-}-\theta\left(-z, z_{0}\right) \xi_{0}^{-} ; \\
& \breve{\xi}_{m}=\theta\left(z, z_{0}\right) \xi_{m}^{+}-\theta\left(z,-z_{0}\right) \xi_{m}^{-}+\theta\left(-z,-z_{0}\right) \xi_{m}^{-}-\theta\left(-z, z_{0}\right) \xi_{m}^{+} \\
& \xi_{n}=\theta(z) \theta\left(z_{0}\right) \xi_{n}^{+}+\theta(-z) \theta\left(-z_{0}\right) \xi_{n}^{-}, R_{j, n}^{0}=\theta\left(z, z_{0}\right) \widehat{R}_{1, n}^{0,+}+\theta\left(-z,-z_{0}\right) \stackrel{\leftarrow}{R}_{1, n}^{0,-},
\end{aligned}
$$

\section{Stress and displacement fields in the plane of connection of}

half-spaces. Putting $z=0$ in the fundamental solutions, we obtain the distribution of normal and tangential stresses and displacements in the plane of connection of half-spaces in the presence of heat sources.

$$
\begin{gathered}
\sigma_{3}(x, y)=-\sum_{n=1}^{3} \frac{R_{1, n}^{0}}{\sqrt{r_{0}^{2}+\left(\xi_{n} z_{0}\right)^{2}}}+\frac{\omega_{1}}{\sqrt{r_{0}^{2}+\left(\breve{\xi}_{0} z_{0}\right)^{2}}}+\sum_{n=1}^{3} \frac{\alpha_{1, n}}{\sqrt{r_{0}^{2}+\left(\breve{\xi}_{n} z_{0}\right)^{2}}}, \\
\sigma_{4}(x, y)=\left(y-y_{0}\right)\left\{\sum_{n=1}^{3} \frac{R_{2, n}^{0}\left(r_{0}^{2}+\left(\xi_{n} z_{0}\right)^{2}\right)^{-1 / 2}}{\left(\xi_{n}\left|z_{0}\right|+\sqrt{r_{0}^{2}+\left(\xi_{n} z_{0}\right)^{2}}\right)}-\frac{\omega_{2}\left(r_{0}^{2}+\left(\breve{\xi}_{0} z_{0}\right)^{2}\right)^{-1 / 2}}{\left(\breve{\xi}_{0}\left|z_{0}\right|+\sqrt{r_{0}^{2}+\left(\breve{\xi}_{0} z_{0}\right)^{2}}\right)}-\right. \\
\left.-\sum_{n=1}^{3} \frac{\alpha_{2, n}\left(r_{0}^{2}+\left(\breve{\xi}_{n} z_{0}\right)^{2}\right)^{-1 / 2}}{\left(\breve{\xi}_{n}\left|z_{0}\right|+\sqrt{r_{0}^{2}+\left(\breve{\xi}_{n} z_{0}\right)^{2}}\right)}\right\},
\end{gathered}
$$




$$
\begin{aligned}
& \sigma_{5}(x, y)=\left(x-x_{0}\right)\left\{\sum_{n=1}^{3} \frac{R_{2, n}^{0}\left(r_{0}^{2}+\left(\xi_{n} z_{0}\right)^{2}\right)^{-1 / 2}}{\left(\xi_{n}\left|z_{0}\right|+\sqrt{r_{0}^{2}+\left(\xi_{n} z_{0}\right)^{2}}\right)}-\frac{\omega_{2}\left(r_{0}^{2}+\left(\breve{\xi}_{0} z_{0}\right)^{2}\right)^{-1 / 2}}{\left(\breve{\xi}_{0}\left|z_{0}\right|+\sqrt{r_{0}^{2}+\left(\breve{\xi}_{0} z_{0}\right)^{2}}\right)}-\right. \\
& \left.-\sum_{n=1}^{3} \frac{\alpha_{2, n}\left(r_{0}^{2}+\left(\breve{\xi}_{n} z_{0}\right)^{2}\right)^{-1 / 2}}{\left(\breve{\xi}_{n}\left|z_{0}\right|+\sqrt{r_{0}^{2}+\left(\breve{\xi}_{n} z_{0}\right)^{2}}\right)}\right\} \\
& u_{1}(x, y)=\left(x-x_{0}\right)\left\{\sum_{n=1}^{3} \frac{R_{3, n}^{0}}{\left(\xi_{n}\left|z_{0}\right|+\sqrt{r_{0}^{2}+\left(\xi_{n} z_{0}\right)^{2}}\right)}-\frac{\omega_{3}}{\left(\breve{\xi}_{0}\left|z_{0}\right|+\sqrt{r_{0}^{2}+\left(\breve{\xi}_{0} z_{0}\right)^{2}}\right)}-\right. \\
& \left.-\sum_{n=1}^{3} \frac{\alpha_{3, n}}{\left(\breve{\xi}_{n}\left|z_{0}\right|+\sqrt{r_{0}^{2}+\left(\breve{\xi}_{n} z_{0}\right)^{2}}\right)}\right\} \\
& u_{2}(x, y)=\left(y-y_{0}\right)\left\{\sum_{n=1}^{3} \frac{R_{3, n}^{0}}{\left(\xi_{n}\left|z_{0}\right|+\sqrt{r_{0}^{2}+\left(\xi_{n} z_{0}\right)^{2}}\right)}-\frac{\omega_{3}}{\left(\breve{\xi}_{0}\left|z_{0}\right|+\sqrt{r_{0}^{2}+\left(\breve{\xi}_{0} z_{0}\right)^{2}}\right)}-\right. \\
& \left.-\sum_{n=1}^{3} \frac{\alpha_{3, n}}{\left(\breve{\xi}_{n}\left|z_{0}\right|+\sqrt{r_{0}^{2}+\left(\breve{\xi}_{n} z_{0}\right)^{2}}\right)}\right\}, \\
& u_{3}(x, y)=-\sum_{n=1}^{3} R_{4, n}^{0}\left(\ln \frac{c}{2}+\ln \left(\left|z_{0}\right| \xi_{n}^{+}+\sqrt{r_{0}^{2}+\left(\xi_{n} z_{0}\right)^{2}}\right)\right)+ \\
& +\omega_{4}\left(\ln \frac{c}{2}+\ln \left(\left(\breve{\xi}_{0}\left|z_{0}\right|\right)+\sqrt{r_{0}^{2}+\left(\breve{\xi}_{0} z_{0}\right)^{2}}\right)\right)+ \\
& +\sum_{n=1}^{3} \alpha_{4, n}\left(\ln \frac{c}{2}+\ln \left(\left(\breve{\xi}_{n}\left|z_{0}\right|\right)+\sqrt{r_{0}^{2}+\left(\check{\xi}_{n} z_{0}\right)^{2}}\right)\right) \text {. }
\end{aligned}
$$

5. Discussion and numerical results. Numerical investigations of the temperature distribution were carried out depending on the thermophysical properties of materials. Figures 1-4 show the temperature distribution in the $Z 0 Y$ plane depending on for some combinations of transversely isotropic materials. In particular, for zinc $Z n:\left(\lambda_{1}^{+}=115, \lambda_{3}^{+}=117, /^{0}\right)$ - material m1; Cadmium $\mathrm{Cd}:\left(\lambda_{1}^{-}=93, \lambda_{3}^{-}=94, /^{0}\right)$ - material m2; aluminum oxide $\mathrm{Al}_{2} \mathrm{O}_{3}:\left(\lambda_{1}^{+}=25 \lambda_{3}^{+}=30 /{ }^{0}\right)$ - $\mathrm{m} 3$ material; magnesium $\mathrm{Mg}:\left(\lambda_{1}^{-}=156, \lambda_{3}^{-}=157, /^{0}\right)$ - material $\mathrm{m} 4$, and in the presence of two heat sources of the same power $Q=10^{5}$

\section{CONCLUSION}

The problem of constructing fundamental solutions to the thermoelasticity problem for a piecewise-homogeneous transversely isotropic space is reduced to the matrix Riemann problem in the space of generalized slow growth functions.As a result of the solution of which, were obtained expressions in explicit 


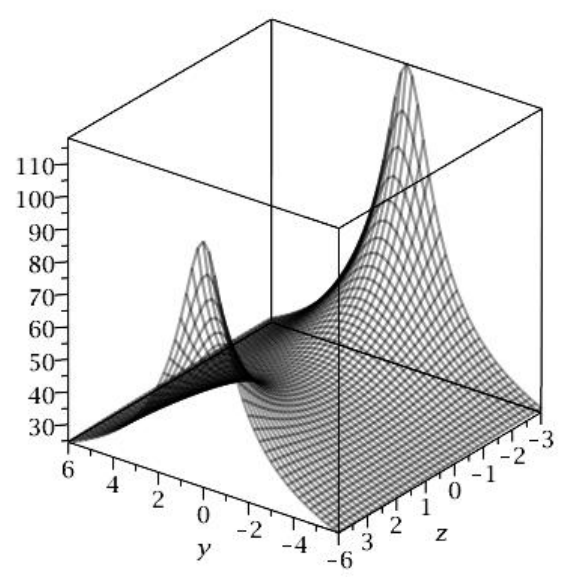

Fig. 1 m1-m2

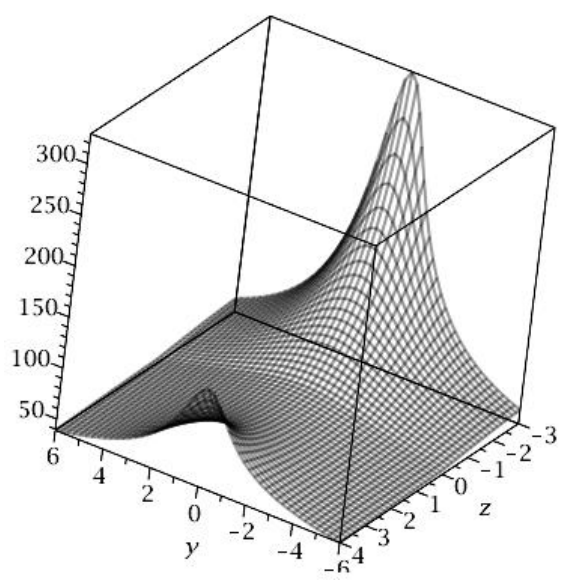

Fig. 3 m2-m3

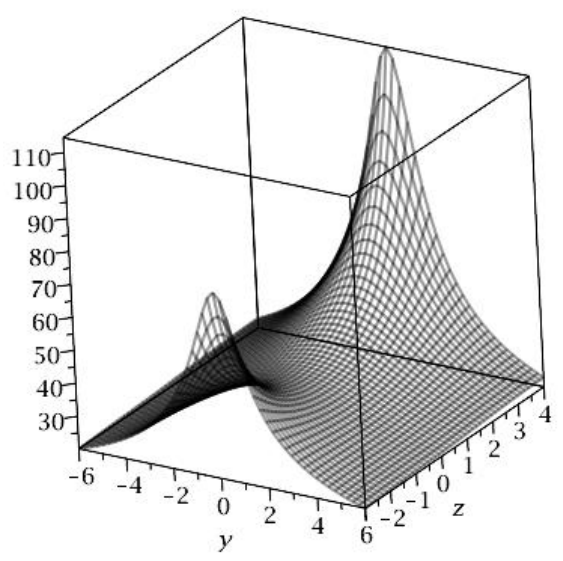

Fig. 2 m2-m4

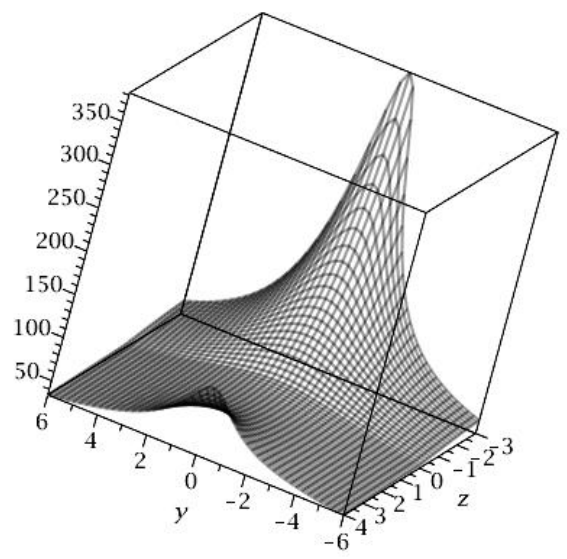

Fig. 4 m1-m3

form for the components of the vector of the fundamental solution of the heat conduction problem, as well as simple representations for the components of the stress tensor and the displacement vector in plane of connection of transversely isotropic elastic half-spaces containing concentrated stationary heat sources. The temperature distribution is investigated depending on the thermophysical characteristics of the half-space materials.

Кривий О. Ф., Морозов Ю. О.

ФУНДАМЕНТАЛЬНІ РОЗВ'ЯЗКИ ЗАДАЧІ ТЕРМОПРУЖНОСТІ ДЛЯ КУСКОВО-ОДНОРІДНОГО ТРАНСВЕРСАЛЬНО-ІЗОТРОПНОГО ПРУЖНОГО ПРОСТОРУ

Резюме 
Проблема побудови фундаментальних розв'язків задачі термопружності для кусковооднорідного трансверсально-ізотропного простору зведена до матричної задачі Рімана в просторі узагальнених функцій повільного зростання. В результаті розв'язування якої отримано в явному вигляді вирази для компонент вектора фундаментального розв'язку задачі теплопровідності, а також прості подання для компонент тензора напружень і вектора переміщень у площині з'єднання трансверсально-ізотропних пружних півпросторів, які містять зосереджені стаціонарні джерелі тепла. Досліджено розподіл температури в залежності від теплофізичних характеристик матеріалів півпросторів.

Ключові слова: фундаментальні розв'язки, матрична задача Рімана, трансверсальноізотропний неоднорідний простір, узагальнені функиій.

Кривой А. Ф., Морозов Ю. А.

ФУНДАМЕНТАЛЬНЫЕ РЕШЕНИЯ ЗАДАЧ ТЕРМОУПРУГОСТИ ДЛЯ КУСОЧНО-ОДНОРОДНОЙ ТРАНСВЕРСАЛЬНО-ИЗОТРОПНОЙ УПРУГОГО ПРОСТРАНСТВА

\section{Резюме}

Проблема построения фундаментальных решений задачи термоупругости для кусочнооднородной трансверсально-изотропного пространства сведена к матричной задачи Римана в пространстве обобщенных функций медленного роста. В результате решения которой получено в явном виде выражения для компонент вектора фундаментального решении задачи теплопроводности, а также простые представления для компонент тензора напряжений и вектора перемещений в плоскости соединения трансверсальноизотропных упругих полупространств, содержащих сосредоточенные стационарные источнике тепла. Исследовано распределение температуры в зависимости от теплофизических характеристик материалов полупространств.

Ключевые слова: фундаментальные решения, матричная задача Римана, трансверсальноизотропное неоднородное пространство, обобщенные функиии.

\section{REFERENCES}

1. Kit H. S., Sushko O. P. Axially symmetric problems of stationary heat conduction and thermoelasticity for a body with thermally active or thermally insulated disk inclusion (crack) // J. Math. Sci. (2011) - 176, No. 4. - P. 561-577. https://doi.org/10.1007/s10958-011-0422-7

2. Kit H. S., Sushko O. P. Problems of stationary heat conduction and thermoelasticity for a body with a heat permeable disk-shaped inclusion (crack) // J. Math. Sci. (2011) 174, No. 3. - P. 309-321. - https://doi.org/10.1007/s10958-011-0422-7

3. Efimov V. V.,Krivoi A. F., Popov G. Ya. Problems on the stress concentration near a circular imperfection in a composite elastic medium // Mech. Solids. (1998) - 33, No. 2. - P. 35-49.

4. Kryvyi O. F. Mutual influence of an interface tunnel crack and an interface tunnel inclusion in a piecewise homogeneous anisotropic space // J. Math. Sci. (2015) - 208, No. 4. - P. 409-416. - https://doi.org/10.1007/s10958-015-2455-9. 
5. Kryvyi O. F. Delaminated interface inclusion in a piecewise homogeneous transversely isotropic space // Mater. Sci. (2014). - 50, No. 2. - P. 245-253. https://doi.org/10.1007/s11003-014-9714-7.

6. Kryvyi O. F. Interface circular inclusion under mixed conditions of interaction with a piecewise homogeneous transversally isotropic space // J. Math. Sci. (2012) - 184, No. 1. - P. 101-119. - https://doi.org/10.1007/s10958-012-0856-6.

7. Kryvyi O. F. Singular integral relations and equations for a piecewise homogeneous transversally isotropic space with interphase defects // J. Math. Sci. (2011) - 176, No. 4. - P. 515-531. - https://doi.org/10.1007/s10958-011-0419-2.

8. Kryvyi O. F., Morozov Yu. O. Solution of the problem of heat conduction for the transversely isotropic piecewise-homogeneous space with two circular inclusions // J. Math. Sci. (2019) - 243, No. 1. - P. 162-182. - https://doi.org/10.1007/s10958-019-04533-1.

9. Kryvyi O. F., Morozov Yu. O. Interphase circular inclusion in a piecewise-homogeneous transversely isotropic space under the action of a heat flux // Proc. 1st Int. conf. Theor. Appl. Exper. Mech., ICTAEM-2018 / E. Gdoutos (ed). - P. 394-396. https://doi.org/10.1007/978-3-319-91989-8-94.

10. Kryvyi O. F. The discontinuous solution for the piece-homogeneous transversal isotropic medium // Oper. Theory: Adv. Appl. (2009) - 191. - P. 395-406. https://doi.org/10.1007/978-3-7643-9921-4-25

11. Kryvyi O. F. Mutual influence of an interface tunnel crack and an interface tunnel inclusion in a piecewise homogeneous anisotropic space / O. F. Kryvyi // J. Math. Sci. (2015) - 208, No. 4. - P. 409-416. - https://doi.org/10.1007/s10958-015-2455-9.

12. Kryvyi O. F. Tunnel internal crack in a piecewise homogeneous anisotropic space // J. Math. Sci. (2014) - 198, No. 1. - P. 62-74. - https://doi.org/10.1007/s10958-014-17737.

13. Kryvyi O. F., Popov G. Ya. Features of the stress field near tunnel inclusions in an inhomogeneous anisotropic space //Int. Appl. Mech. (2008) - 44, No. 6. - P. 626-634.https://doi.org/10.1007/s10778-008-0084-4.

14. Kryvyi O. F.,Popov G. Ya., Radiollo M. V. Certain problems of an arbitrarily oriented stringer in a composite an isotropic plane //J. Appl. Math. Mech. (1986) - 50, No. 4. P. 475-483. - https://doi.org/10.1016/0021-8928(86)90012-2.

15. Kryvyi O. F., Morozov Yu. O. Thermally active interphase inclusion in a smooth contact conditions with transversely isotropic half-spaces // Frattura ed Integrita Strutturale, 52 (2020) 33-50; DOI: 10.3221/IGF-ESIS.52.04.

16. Yue Z. Q. Elastic fields in two joined transversely isotropic solids due to concentrated forces // Int. J. Eng. Sci. (1995) - 33, No. 3. - P. 351-369.https://doi.org/10.1016/0020-7225(94)00063-P.

17. Li X.F., Fan T.Y. The asymptotic stress field for a ring circular inclusion at the interface of two bonded dissimilar elastic half-space materials // Int. J. Solids Struct. (2001) - 38, No. 44-45. - P. 8019-8035. - https://doi.org/10.1016/S0020-7683(01)00010-5. 
18. Hou P.F., Leung A. T. Y., He Y.J. Three-dimensional Green's functions for transversely isotropic thermoelastic bimaterials // Int. J. Solids Struct. (2008) - 45, No. 24 .- P. 6100-6113. - doi.org/10.1016/j.ijsolstr. 2008.07.022.

19. Kumar R., Gupta V. Green's function for transversely isotropic thermoelastic diffusion bimaterials // J. Therm. Stresses. (2014) - 37, No. 10. - P. 1201-1229.

20. Kushnir R. M., Protsyuk Yu. B. Thermoelastic state of layered thermosensitive bodies of revolution for the quadratic dependence of the heat-conduction coefficients // Mater Sci. (2011) - 46, No. 1. - P. 1-15. - https://doi.org/10.1007/s11003-010-9258-4.

21. Kushnir R. M., Protsyuk B. A. A method of the Green's functions for quasistatic thermoelasticity problems in layered thermosensitive bodies under complex heat exchange // Oper. Theory: Adv. Appl. (2009) - 191. - P. 143-154. - doi.org/10.100 7/978-37643-9921-4-9. 